\title{
Using films as a psychoeducation tool for patients with schizophrenia: a pilot study using a quasi-experimental pre-post design
}

Christian von Maffei ${ }^{1}$, Frauke Görges ${ }^{1}$, Werner Kissling ${ }^{3}$, Wolfgang Schreiber ${ }^{4}$ and Christine Rummel-Kluge ${ }^{1,2^{*}}$

\begin{abstract}
Background: Relapses and, subsequently, readmissions are common in patients with schizophrenia. Psychoeducation has been shown to reduce the number and duration of readmissions. Yet, only little more than $20 \%$ of psychiatric patients in German speaking countries receive psychoeducation. Among other reasons, costs may be considered too high by hospitals. The objective of the present study was to test the feasibility of a new cost-efficient approach in the psychoeducation of patients with schizophrenia. In this study, films were used to impart knowledge about the illness to inpatients.
\end{abstract}

Methods: A total of 113 participants were initially included in the study, eleven of which were not included in the final analyses. Six films about the symptoms, diagnosis, causes, warning signs, treatment of schizophrenia and about the influence of family members and friends were shown in a group setting in the presence of nursing staff. All films combined facts, expert opinions, and personal experiences of peers. As the main outcome criterion of this feasibility pilot study, we measured the effects on knowledge. Secondary outcome measures included compliance, insight into illness, side effects, and quality of life. Data were collected directly after the intervention and about half a year afterwards. The number and the duration of readmissions to the hospital were recorded and compared to the number and duration of prior admissions. Patients were also asked to state their subjective opinion about the films. Main data analyses were done using paired t-tests and Wilcoxon signed-rank tests. Secondary analyses also involved ANOVAs and ANCOVAs.

Results: One hundred and two inpatients were included in the data analyses. Showing the films in the tested setting was shown to be feasible. Knowledge about schizophrenia $(p<.001)$, compliance $(p s<.01)$, insight into illness $(p<.01)$, and quality of life $(p<.001)$ all increased significantly after patients had watched the films and remained stable for at least half a year. A vast majority (84.9\%) of the patients found the films to be interesting and informative.

Conclusions: Using films to educate inpatients about schizophrenia is a feasible method that is cost- and time-efficient and well received by the patients.

Keywords: Schizophrenia, Psychoeducation, Film, Group

\footnotetext{
* Correspondence: christine.rummel-kluge@medizin.uni-leipzig.de

'Department of Psychiatry and Psychotherapy, University of Leipzig,

Semmelweisstr. 10, D-04103 Leipzig, Germany

${ }^{2}$ Forschungszentrum Depression der Stiftung Deutsche Depressionshilfe,

Semmelweisstr. 10, 04103 Leipzig, Germany

Full list of author information is available at the end of the article
} 


\section{Background}

Schizophrenia is a disease that often takes a chronic course. Relapses with and without readmission to the hospital are common and often associated with medication non-compliance [1-8]. There are various causes for non-compliance, including informational deficits, consequences of psychopathological symptoms, such as cognitive impairments or poor insight into illness, subjective response to medication, and side effects [9-12].

Relapses come at a high price for both the patient and the health care system. The relationship between noncompliance and increased costs for treatment has become apparent in previous research $[6,13,14]$. Different approaches have been taken to improve compliance and to thereby reduce the costs of (partial) non-compliance, several of which have been proven to be effective [15]. One promising approach is psychoecucation [16]. Researchers found positive effects of psychoeducative programs on knowledge, compliance, psychopathology, satisfaction with treatment, and global functioning. They were also related to a change of illness concept and to fewer relapses as well as fewer and shorter readmissions to the hospital [17-20].

In a large-scale randomized multicenter study involving 236 inpatients (Psychosis Information Project Study), Pitschel-Walz et al. [21] investigated the long-term effects of a short psychoeducative program on the number and duration of readmissions, compliance (rated by treating physician) and global functioning (GAF) 12 and 24 months after index hospitalization. The program comprised 8 one-hour group sessions, in which patients received information about symptoms, etiology, treatment, and relapse prevention. Close relatives received a similar group intervention. For all the outcome variables, the authors demonstrated positive effects of psychoeducation on the intervention group compared to a control group, who received treatment as usual. An increase in knowledge and an improvement in illness concept were also observed in a 6-month follow-up questionnaire [22]. Despite the great number of studies demonstrating the effectiveness of psychoeducative programs and calls for them to be a part of standard treatment [16], this form of intervention is still not implemented across the board.

According to a study investigating the implementation of psychoeducation for schizophrenia, in 2003, programs were offered by $83 \%$ of hospitals in Germany, Austria, and Switzerland. However, overall, only $21 \%$ of the patients received psychoeducation. Dropout was high with a rate of $25 \%$ [23,24]. Several factors may have contributed to this situation. Some hospitals may still question the effectiveness of such programs, but most of the hospitals simply do not have enough staff to provide a wellprepared weekly psychoeducation program for their patients. And even for those who do, reaching their patients seems to be a difficult task. In some patients, symptoms may be too severe. Others are discharged (with or against medical advice) before they complete the program, and some patients lack the motivation to join or complete the program. While, from the hospital's and the patient's point of view, many of these reasons for not offering or participating in psychoeducation are understandable, the costs are high. Rummel-Kluge et al. estimated that up to 150 million Euros could be saved each year by tripling the number of patients receiving psychoeducation [23].

The aim of the pilot study reported here was to investigate the feasibility of a short, time- and cost-efficient tool for psychoeducation, which would also be wellreceived by patients. To tackle the issue of psychoeducation being too time consuming and expensive, we used a program consisting of six films, in which the main topics concerning schizophrenia and its treatment are covered [17]. The patients' motivation was addressed by making the films shorter than the usual one-hour program and by presenting facts more vividly by backing them up through expert opinions and by adding stories of other patients' personal experiences. Previous research showed that peers as counsellors and educators are very well received by psychiatric patients [25-27]. In a quasi-experimental pre-post design, several measures were assessed. As the main outcome variable, we measured the patients' knowledge about their illness before and after the intervention. Secondary outcome variables included the number and duration of readmissions, compliance, insight into illness, side effects, and quality of life. Most of these measures were assessed before, immediately after the intervention, and approximately half a year after the intervention. Compliance was measured before and half a year after the intervention. Readmission data were taken from the hospital data base. In addition, patients were asked to state their personal opinion on the content and on the interestingness of the films. Both objective and subjective measures were used to gain insight into the feasibility of this new approach.

\section{Methods}

\section{Intervention}

Six films of about 17 minutes in length each were used for this psychoeducative program. Originally, the films were part of a larger psychoeducation program (Alliance Psychoeducation Program [17]). The script was written by two of the authors of the present study and the films were produced by a professional company. The films were very well received by patients who participated in the evaluation of the Alliance Psychoeducation Program, leading up to the idea to use the films standalone. They included objective information about schizophrenia given by a presenter. Objective information was accompanied by clips in which patients gave statements about their personal experiences. Information about the treatment of schizophrenia was given by experts. Films were presented 
continuously in a fixed order (1. symptoms of schizophrenia, 2. diagnosis of schizophrenia and dealing with schizophrenia, 3. causes of schizophrenia, 4. medication effect and side effects, 5 . warning signs before relapse, 6 . influence of family members and friends) with each of the cycles lasting two weeks. On average, patients watched three films per week. They could enter the program at any point, starting with the film that was presented next. A member of the nursing staff was always present, but did not actively interfere with the program. However, the nurse was allowed to respond to questions. At the end of each session, patients were asked to give a short, written evaluation of the film. A session lasted for about 35 minutes, including the evaluation.

Patients received standard psychiatric care with psychopharmacological treatment. Written informed consent from each patient and institutional review board approval were obtained (Ethics Committee of the Faculty of Medicine, Technische Universität München, Germany).

\section{Participants}

A total of 113 patients from a psychiatric hospital in Mainkofen, Germany were initially included in the study. Patients were included if they met the following criteria: a) aged 18-65, b) diagnosis of schizophrenia or schizoaffective disorder according to ICD-10, c) according to the clinical decision of the treating physician, psychotic symptoms had decreased enough to be able to follow psychoeducative content and to fill out questionnaires, d) no signs of organic brain damage or intellectual disability, e) adequate knowledge of the German language, $\mathrm{f}$ ) adequate eye sight, and g) estimated duration of stay of at least two weeks. Participants were included in the data analyses if they had watched at least five of the six films. Eleven patients did not meet the criteria (e.g. due to change in diagnosis) and had to be excluded from data analyses.

\section{Assessment}

There were two main measuring points for data assessment: immediately before the intervention (T0) and immediately after the intervention ( $\mathrm{T} 1$; on average 15 days after the first assessment). In addition, it was possible to obtain data from some patients and their physicians a few months after the intervention to assess the stability of the effects (T2; for patients on average 150 days and for doctors on average 163 days after the first assessment).

\section{Self-assessment}

The Knowledge of Illness about Schizophrenia Questionnaire [28] was used to assess a potential increase in knowledge. It is an instrument for assessing knowledge of patients with schizophrenia about symptoms, causes, treatment, and warning signs. It comprises 21 questions with 107 single items. The maximum score is 70 points. The questionnaire is reliable (reliability coefficient $\alpha=.91)[19,29]$.

In order to assess the adherence to medication, the Medication Adherence Rating Scale (MARS) was used. The MARS comprises ten items. The score ranges from 0 to 10 points with 10 points indicating more compliance. The MARS shows high internal consistency with a Cronbach's alpha of .75 [30].

In addition, single items were used to assess how well the patient felt informed about schizophrenia (4-point scale: 1 - very good, 2 - good, 3 - moderate, 4 - poor), perceived quality of life (5-point scale: 1 - very poor, $2-$ poor, 3 - average, 4 - good, 5 - very good), contentment with health (5-point scale: 1 - very discontent, 2 - discontent, 3 - neither content nor discontent, 4 - content, 5 - very content), and the subjective rating of the strength of medication side effects (Visual Analogue Scale with 0 indicating strong side effects and 100 indicating no side effects).

\section{External assessment}

All external assessments for T0 and T1 were done by nurses from the hospital. External assessments for T2 were done by the psychiatrist/neurologist or general practitioner, from whom the patient received outpatient treatment at the time. The physicians evaluating T2 measures were blind to the nurses' assessment at T0 and T1. Compliance was only measured at $\mathrm{T} 0$ and $\mathrm{T} 2$, not at $\mathrm{T} 1$.

Compliance was rated on a 4-point scale that was exactly operationalized ( 1 - very good, 2 - good, $3-\bmod$ erate, 4 - poor; see Pitschel-Walz et al. [21] for exact operationalization) and insight into the illness was rated on a 3-point scale (1 - insightful, 2 - somewhat insightful, 3 not insightful; fourth alternative: not relevant). This item was taken from the Scale to Assess Unawareness of Mental Disorder. Inter-rater ICC for the item was .89 [31].

Frequency and duration of (re-)admissions to the hospital were obtained from the hospital data base for 70 weeks prior to the index admission and for 70 weeks after index discharge.

Immediately after watching a film, participants were presented with three statements and asked to check a box if they agreed with them. The statements were the following: "It was interesting and informative.", "I already knew most of the content.", "I was too troubled by this". In addition, they were asked to write down what they had liked most and what had helped them most.

\section{Statistical analysis}

Descriptive methods were used for statistical analyses of sociodemographic and feedback data from participants. For Visual Analogue Scales and summed scores, such as the Knowledge of Illness score and the MARS score as 
well as the subjective strength of side effects, equidistance and normality [32] were assumed. These comparisons were made using paired $t$-tests. For our primary outcome measure, the $95 \%$ confidence interval of the difference was analyzed. Comparisons involving all other data were made using the Wilcoxon signed-rank test. Exploratory analyses, which are reported in the discussion, were made using ANOVAs to test for an interaction of prior treatment with the increase in knowledge and ANCOVAs to test for the influence of perceived side effects on compliance. All calculations were done with PASW statistics, versions 18.0.0 and 20.0.0.2.

\section{Results}

\section{Reception of films}

Overall, the films were well received by the patients. On average, $84.9 \%$ of the participants thought them to be interesting and informative. To an average of $82.3 \%$, most of the content of the films was new. Only 7.5\% thought that the films were too upsetting to watch, while $92.5 \%$ did not think so.

\section{Pre-post comparisons}

Sociodemographic data are presented in Table 1. Means, standard deviations, and sample sizes for each dependent measure are given in Table 2.

\section{Self-assessment}

There was a significant increase of 7.3 points in the knowledge of illness score, $t(91)=-9.87, p<.001$. The $95 \% \mathrm{CI}$ $[5.8,8.8]$ of the difference between the two means did not include the value " 0 " and therefore corroborates the finding of a significant increase in our primary outcome measure.

Patients also scored higher in the MARS, $t(72)=-3.90$, $p<.001$, after they had participated in the psychoeducation program. They felt more informed about schizophrenia, $Z=-6.52, p<.001$, rated quality of life higher, $Z=-4.70, p<.001$, and felt more content with their health, $Z=-4.21, p<.001$. The subjective rating of the strength of medication side effects did not decrease $t<1$.

\section{External assessment}

There was a significant increase in the rating of insight into illness, $Z=-2.90, p<.01$. However, neither frequency nor duration of (re-)admissions to the hospital changed significantly after the intervention, all $p \mathrm{~s}>.140$.

\section{Long-term effects}

Data for T2 are available from only about half of the patients. Thus, they were analyzed separately and should be interpreted carefully.

Compliance, as rated by nurses and doctors, increased significantly, $Z=-2.62, p<.01$. The knowledge
Table 1 Sociodemographic data of participants, $n=102$

\begin{tabular}{lll}
\hline Characteristic & M & SD \\
\hline Age (years) & 35.1 & 9.0 \\
Duration of Stay (days) & 57.4 & 26.6 \\
Duration of Illness (years) & 11.1 & 10.6 \\
Number of prior hospitalizations* $^{*}$ & 3.8 & 4.0 \\
\hline & $\mathbf{n}$ & $\%$ \\
\hline
\end{tabular}

Gender

Male

$69 \quad 67.6$

Female

$33 \quad 32.4$

Diagnosis

Schizophrenia

Schizoaffective Disorder

$86 \quad 84.3$

Pharmacological Treatment

$\begin{array}{lll}\text { Atypical Antipsychotic } & 74 & 72.5\end{array}$

$\begin{array}{lll}\text { Typical Antipsychotic } & 15 & 14.7\end{array}$

Both

$13 \quad 12.7$

Prior participation in a psychoeducation program $\begin{array}{lll}\text { Yes } & 16 & 15.7\end{array}$

$\begin{array}{lll}\text { No } & 85 & 83.3\end{array}$

n/a

$1 \quad 1$

Employment status

$\begin{array}{lll}\text { Unemployed } & 26 & 25.5\end{array}$

In training

Competitive employment

$6 \quad 5.9$

Non-competitive employment

$23 \quad 22.6$

retired

$6 \quad 5.9$

n/a

Living status

Independently

With parents/siblings/relatives

With partner/children

In a rehabilitation facility

*Information was available from 96 patients

of illness score was still elevated at T2 when compared to T0, $t(52)=-4.35, p<.001$ and did not differ from $\mathrm{T} 1, t(52)=0.88, p=.384(\mathrm{n}=53)$. The same pattern was found for the MARS score, T0-T2: $t(42)=-2.14, p=.038$ and $t(42)=0.15, p=.883$ respectively $(\mathrm{n}=43)$, perceived quality of life, $Z=-2.27, p<.05$ and $Z=-0.66, p=.509$ respectively $(\mathrm{n}=51)$, contentment with health, $Z=-1.99$, $p<.05$ and $Z=-0.62, p=.533$ respectively $(\mathrm{n}=51)$, and insight into illness, $Z=-2.32, p<.05$ and $Z=-0.57$, $p=.567$ respectively $(\mathrm{n}=49)$.

Patients still felt more informed about schizophrenia at T2 when compared to T0, $Z=-4.83, p<.001$. There was, however, a small decrease from $\mathrm{T} 1$ to $\mathrm{T} 2, Z=-1.96$, $p<.05(\mathrm{n}=54)$. Again, there were no effects for the 
Table 2 Means and standard deviations (in Parentheses) for T0 and T1, as well as sample sizes for each measure

\begin{tabular}{|c|c|c|c|}
\hline Measure & T0 & $\mathrm{T1}$ & $\mathrm{n}^{*}$ \\
\hline Knowledge of Illness about schizophrenia questionnaire & $41.1(14.2)$ & $48.4(14.2)$ & 92 \\
\hline MARS & $7.2(2.0)$ & $7.9(1.8)$ & 73 \\
\hline Insight into Illness & $1.6(0.7)$ & $1.4(0.6)$ & 91 \\
\hline Feeling of being informed about schizophrenia & $2.7(0.9)$ & $1.8(0.7)$ & 94 \\
\hline Subjective strength of side effects & $65.2(28.1)$ & $65.8(25.8)$ & 90 \\
\hline Perceived quality of life & $3.0(1.0)$ & $3.5(0.9)$ & 92 \\
\hline \multirow[t]{2}{*}{ Contentment with health } & $3.0(1.2)$ & $3.5(1.0)$ & 91 \\
\hline & Pre & Post & $\mathrm{N}^{*}$ \\
\hline Compliance & $2.2(1.0)$ & $1.7(1.0)$ & 54 \\
\hline Frequency of (re-)admission & $0.5(1.0)$ & $0.6(0.9)$ & 101 \\
\hline Duration of (re-)admission & $22.8(49.4)$ & $26.3(42.2)$ & 101 \\
\hline
\end{tabular}

Significant results are indicated by bold text.

*Data were not available from every patient for each outcome measure. As many data points as possible were included in each analysis, causing the sample size to differ between outcome measures.

subjective rating of the strength of medication side effects, $t<1(\mathrm{n}=54)$.

\section{Discussion}

The present study aimed at investigating the feasibility of a new approach for psychoeducation that is more timeand more cost-efficient compared to a standard psychoeducation program, while integrating vital information about schizophrenia given by professionals with the personal experience of peers, who patients can relate to. The intervention proved to be effective in several measures.

\section{Knowledge about schizophrenia increased}

Patients were more informed about their illness at the end of the psychoeducation program and seemed to still remember this information after a few months had passed. Subjectively, patients also felt more informed about schizophrenia immediately after the intervention, but seemed to feel somewhat less confident after a few months. This effect might be reduced if patients had the opportunity to visit booster sessions or if the films were made available for private use, giving further access to information and to emotional support after being discharged. The two-month period directly following a hospitalization is especially important for adherence as the readmission rate is particularly high here [33]. Being able to watch the films at home may also aid those patients, who were not able to complete the program at the hospital due to early discharge and, as a desirable side effect, this also raises the likelihood of relatives watching the films. The importance of educating relatives has been shown in many studies, however, it is still not being done thoroughly in severe mental illnesses, where stigma and social avoidance remain a serious problem [16,34-37].

\section{Compliance and insight into illness increased}

Compliance was also higher after the intervention as indicated by the self-assessment (MARS) and external assessment (rated by nurses and doctors). The increase in compliance seemed to be long-term. Alongside compliance, insight into illness, which might be a crucial prerequisite, also increased. Unfortunately, the number of (re-)admissions did not decrease in the first 70 weeks after index discharge. However, schizophrenia often takes a chronic course making readmissions a necessity to protect the patients from the consequences of their disease. Thus, readmissions, especially if they happen voluntarily and early during a relapse, can be considered a success. This notion is supported by the increase in the quality of life ratings. Although the patients spent about as much time in the hospitals as they had done before the index treatment, they were more content with their health and perceived a higher quality of life.

However, compliance may have increased because the medication started to work, thereby also reducing the consequences of psychopathological symptoms, such as impaired cognitive functions and impaired insight into illness. However, patients were only included in the study if they were able to follow the content of the films and to fill out questionnaires, implying the ability to be compliant. In addition, the Clinical Global Impression Severity score did not change significantly between T0 $(M=5.9$, $S D=0.7)$ and $\mathrm{T} 1(M=5.8, S D=0.8), p=.354$. The patients' attitude towards their medication may have been better due to a better adjustment of that medication at the end of treatment, causing fewer side effects. However, the perceived strength of side effects did not differ between any of the times of measurement. In an ANCOVA with the perceived strength of side effects at $\mathrm{T} 1$ as covariate, the covariate only influenced the MARS score at a trend level, $F(1,69)=3.25, p=.076, M S E=6.14$, and did 
not influence compliance or insight into illness at all, $F<1$ and $F(1,84)=1.93, p=.168, M S E=0.58$ respectively. For the MARS score, the difference between T0 and $\mathrm{T} 1$ remained significant after controlling for the perceived strength of side effects, $F(1,70)=13.53, p<.001$, $M S E=1.10$. Future studies, however, should also evaluate cognition, which can be an important factor in knowledge acquisition.

\section{Films were well accepted}

There is evidence that insight into a severe mental illness is also associated with negative implications such as self-stigma, shame or lower quality of life [38-40]. The films might have had a similar effect on the patients in the present study. However, our results suggest that any negative effect, if present at all, could not have lasted long. We would have expected more patients to find the films upsetting, which was not the case. Also, perceived quality of life increased in the present study. Any negative effect of gaining insight through the films may have been counteracted by the combination of providing facts and personal experiences of successfully treated patients in the films.

\section{First and multiple episode patients benefit from the intervention}

Both, first time patients $(\mathrm{n}=63 ; t(62)=-8.74, p<.001)$ and others $(\mathrm{n}=29, t(28)=-4.84, p<.001)$ showed a significant increase in knowledge. The interaction of prior treatment with time of measurement for the knowledge of illness score did not exceed a trend level, $F(1,90)=$ $3.15, p=.079, M S E=24.84)$. Importantly, this result indicates that psychoeducation is an effective tool not only during a patient's first episode, but also after one or more relapses.

\section{Limitations}

There are certain limitations to this study, the most obvious one being the use of a quasi-experimental design. Due to the lack of a control group that did not receive the same treatment as the intervention group, we cannot rule out beyond any doubt the possibility that our results were in fact caused by something other than the intervention. For instance, it seems possible that treatment as usual alone may have caused the effects reported above. Patients may have learned about schizophrenia from the hospital staff or from other patients. While this is probably true to some extent, knowledge would have been acquired in a less systematic way, causing variance to be bigger and effects to be smaller. In a previous study, Pitschel-Walz et al. [22] compared the increase in knowledge between an intervention group that participated in a psychoeducative group program and a control group that received treatment as usual. An increased score in the Knowledge of Illness about Schizophrenia Questionnaire was observed for the intervention group only, not for the control group. Taking these results into consideration, it seems highly unlikely that treatment as usual was the cause of the effects observed in the present study. In addition, we would have expected first time patients to have a much higher increase in knowledge than patients who had been treated for schizophrenia in a hospital before. This was not the case. Furthermore, we cannot rule out the possibility that choosing a mixed diagnoses sample may have had an influence on the results. The program was originally planned for patients with a diagnosis of schizophrenia. Patients who suffer from schizoaffective disorder differ in some aspects from patients with schizophrenia and may have responded differently to the content of the films. Unfortunately, the sample of patients with schizoaffective disorder was too small to be analyzed separately. Future research needs to determine the effect of the films on this group of patients.

\section{Conclusions}

Using films to educate patients about schizophrenia and schizoaffective disorder is a cost-saving and time-efficient approach, which was well received by the vast majority of our patients. We were able to show positive effects on knowledge, compliance, insight into illness, and quality of life after the intervention. Both the short-term and longterm effects observed here are promising. However, the findings need to be confirmed by future research and tested against a suitable control group. Future research also needs to determine whether psychoeducation via film is just as effective as other methods, such as standard group psychoeducation led by an expert or a peer. Psychoeducation via film could be a useful tool in different situations. For instance, it could be used in hospitals that are short of staff. Patients could start the program at the hospital and continue watching the films after discharge, or watch them again later. Relatives would get the chance of receiving information about schizophrenia, even when there are no groups offered for them. In addition, these films could be used to educate new staff. Thus, films could be a versatile addition to the standard treatment, especially in view of scarce resources.

\section{Abbreviations}

ANOVA: Analysis of variance; ANCOVA: Analysis of covariance; Cl: Confidence interval; MARS: Medication Adherence Rating Scale.

\section{Competing interests}

The authors declare that they have no competing interests.

\section{Authors' contributions}

CvM contributed to the study design, performed the acquisition of data and the data management. FG and CVM performed the statistical analyses and wrote the paper. CRK conceptualised and coordinated the study, interpreted the data and revised the paper. WK and WS contributed to the study design 
and revised the paper for important intellectual content. All authors read and approved the final manuscript.

\section{Acknowledgements}

We acknowledge support from Leipzig University within the program of Open Access Publishing and we wish to thank the patients and the nursing staff who participated in this study.

\section{Author details}

${ }^{1}$ Department of Psychiatry and Psychotherapy, University of Leipzig Semmelweisstr. 10, D-04103 Leipzig, Germany. ${ }^{2}$ Forschungszentrum Depression der Stiftung Deutsche Depressionshilfe, Semmelweisstr. 10, 04103 Leipzig, Germany. ${ }^{3}$ Department of Psychiatry and Psychotherapy, Technische Universität München, Möhlstr. 26, 81675 München, Germany. ${ }^{4}$ Bezirksklinikum Mainkofen, 94469 Deggendorf, Germany.

\section{Received: 20 November 2014 Accepted: 23 April 2015} Published online: 30 April 2015

\section{References}

1. Schooler NR. Relapse and rehospitalization: comparing oral and depot antipsychotics. J Clin Psychiatry. 2003;64 Suppl 16:14-7.

2. Kissling W, Leucht S. Results of treatment of schizophrenia: is the glass half full or half empty? Int Clin Psychopharmacol. 1999;14 Suppl 3:S11-4.

3. Lacro JP, Dunn LB, Dolder CR, Leckband SG, Jeste DV. Prevalence of and risk factors for medication nonadherence in patients with schizophrenia: a comprehensive review of recent literature. J Clin Psychiatry. 2002;63:892-909.

4. Jeffreys SE, Harvey CA, McNaught AS, Quayle AS, King MB, Bird AS. The Hampstead Schizophrenia Survey 1991. I: Prevalence and service use comparisons in an inner London health authority, 1986-1991. Br J Psychiatry. 1997;170:301-6.

5. Weiden P, Glazer W. Assessment and treatment selection for "revolving door" inpatients with schizophrenia. Psychiatr Q. 1997;68:377-92.

6. Gilmer TP, Dolder CR, Lacro JP, Folsom DP, Lindamer L, Garcia P, et al. Adherence to treatment with antipsychotic medication and health care costs among Medicaid beneficiaries with schizophrenia. Am J Psychiatry. 2004;161:692-9.

7. Rummel-Kluge C, Schuster T, Peters S, Kissling W. Partial compliance with antipsychotic medication is common in patients with schizophrenia. Aust N Z J Psychiatry. 2008;42:382-8.

8. Gibson S, Brand SL, Burt S, Boden ZVR, Benson O. Understanding treatment non-adherence in schizophrenia and bipolar disorder: a survey of what service users do and why. BMC Psychiatry. 2013;13:153.

9. Rettenbacher MA, Hofer A, Eder U, Hummer M, Kemmler G, Weiss EM, et al. Compliance in schizophrenia: psychopathology, side effects, and patients' attitudes toward the illness and medication. J Clin Psychiatry. 2004;65:1211-8.

10. Fenton WS, Blyler CR, Heinssen RK. Determinants of medication compliance in schizophrenia: empirical and clinical findings. Schizophr Bull. 1997;23:637-51.

11. Anselmetti S, Bechi M, Bosia M, Quarticelli C, Ermoli E, Smeraldi E, et al. Theory'of mind impairment in patients affected by schizophrenia and in their parents. Schizophr Res. 2009;115:278-85.

12. Nuechterlein $\mathrm{KH}$, Ventura J, Subotnik KL, Bartzokis G. The early longitudinal course of cognitive deficits in schizophrenia. J Clin Psychiatry. 2014;75:25.

13. Thieda P, Beard S, Richter A, Kane J. An economic review of compliance with medication therapy in the treatment of schizophrenia. Psychiatr Serv. 2003:54:508-16.

14. Weiden PJ, Olfson M. Cost of relapse in schizophrenia. Schizophr Bull. 1995;21:419-29.

15. Nose M, Barbui C, Gray R, Tansella M. Clinical interventions for treatment non-adherence in psychosis: meta-analysis. Br J Psychiatry. 2003;183:197-206.

16. Bauml J, Frobose T, Kraemer S, Rentrop M, Pitschel-Walz G. Psychoeducation: a basic psychotherapeutic intervention for patients with schizophrenia and their families. Schizophr Bull. 2006;32 Suppl 1:S1-9.

17. Rummel-Kluge C, Pitschel-Walz G, Kissling W. A fast, implementable psychoeducation program for schizophrenia. Psychiatr Serv. 2007:58:1226.

18. Pekkala E, Merinder L. Psychoeducation for schizophrenia. Cochrane Database Syst Rev. 2011;15(6):CD002831.

19. Pitschel-Walz G. Die Einbeziehung der Angehörigen in die Behandlung schizophrener Patienten und ihr Einfluss auf den Krankheitsverlauf. In: Lang P, editor. [Europäische Hochschulschriften. Reihe VI, Psychologie Publications universitaires européennes. Série VI, Psychologie European university studies. Series VI, Psychology, vol. 581], vol. 19. Frankfurt am Main, New York; 1997. p. 99-118.

20. Xia J, Merinder LB, Belgamwar MR. Psychoeducation for schizophrenia. Cochrane Database Syst Rev. 2011.15;(6):CD002831.

21. Pitschel-Walz G, Bauml J, Bender W, Engel RR, Wagner M, Kissling W. Psychoeducation and compliance in the treatment of schizophrenia: results of the Munich psychosis information project study. J Clin Psychiatry. 2006;67:443-52.

22. Pitschel Walz G, Boerner R, Mayer CER, Peuker I, Welschehold M, Bender W, et al. Informationszentrierte gruppen für schizophrene patienten und deren angehörige: einfluß von wissen, krankheitskonzept und expressed emotions (PIP-studie). In: Bender W, Hubmann W, Mohr F, editors. Neuere entwicklungen in der behandlung schizophrener psychosen. München-Haar: VTS-Verlag; 1995.

23. Rummel-Kluge C, Pitschel-Walz G, Bauml J, Kissling W. Psychoeducation in schizophrenia-results of a survey of all psychiatric institutions in Germany, Austria, and Switzerland. Schizophr Bull. 2006;32:765-75.

24. Rummel-Kluge C, Kluge M, Kissling W. Frequency and relevance of psychoeducation in psychiatric diagnoses: results of two surveys five years apart in German-speaking European countries. BMC Psychiatry. 2013;13:170

25. Rummel-Kluge C, Stiegler-Kotzor M, Schwarz C, Hansen W, Kissling W. Peer-counseling in schizophrenia: patients consult patients. Patient Educ Couns. 2008;70:357-62

26. McGill CW, Patterson CJ. Former patients as peer counselors on locked psychiatric inpatient units. Psychiatr Serv. 1990;41:1017-9.

27. Rummel CB, Hansen W, Helbig A, Pitschel-Walz G, Kissling W. Peer-to-peer psychoeducation in schizophrenia: a new approach. J Clin Psychiatry. 2005;66:1580-5.

28. Bäuml J, Pitschel-Walz G. Psychoedukation bei schizophrenen Erkrankungen: Konsensuspapier der Arbeitsgruppe "Psychoedukation bei schizophrenen Erkrankungen". Stuttgart, New York: Schattauer; 2003.

29. Froböse T, Bäuml J, Jahn T, Pohl C, Pitschel-Walz G. Knowledge gain by psychoeducation for patients with schizophrenia: associations with sociodemographic data, psychopathology, insight and global neurocognitive performance. Int J Clin Psychiatry Mental Health. 2014;2:111-21.

30. Thompson K, Kulkarni J, Sergejew AA. Reliability and validity of a new medication adherence rating scale (MARS) for the psychoses. Schizophr Res. 2000;42:241-7.

31. Amador XF, Strauss DH, Yale SA, Flaum MM, Endicott J, Gorman JM Assessment of insight in psychosis. Am J Psychiatry. 1993;1:873.

32. Hays WL. Statistics for the social sciences. 2nd ed. New York: Holt, Rinehart and Winston; 1973.

33. Markowitz M, Karve S, Panish J, Candrilli SD, Alphs L. Antipsychotic adherence patterns and health care utilization and costs among patients discharged after a schizophrenia-related hospitalization. BMC Psychiatry. 2013;13:246.

34. McFarlane WR, Dixon L, Lukens E, Lucksted A. Family psychoeducation and schizophrenia: a review of the literature. J Marital Fam Ther. 2003;29:223-45.

35. Cohen AN, Glynn SM, Murray-Swank AB, Barrio C, Fischer EP, McCutcheon SJ, et al. The family forum: directions for the implementation of family psychoeducation for severe mental illness. Psychiatr Serv. 2008;59:40-8

36. Frank F, Rummel-Kluge C, Berger M, Bitzer EM, Holzel LP. Provision of group psychoeducation for relatives of persons in inpatient depression treatment-a cross-sectional survey of acute care hospitals in Germany. BMC Psychiatry. 2014;14:143.

37. Farrelly S, Clement S, Gabbidon J, Jeffery D, Dockery L, Lassman F, et al. Anticipated and experienced discrimination amongst people with schizophrenia, bipolar disorder and major depressive disorder: a cross sectional study. BMC Psychiatry. 2014;14:157.

38. Hasson-Ohayon I, Avidan-Msika M, Mashiach-Eizenberg M, Kravetz S, Rozencwaig S, Shalev H, et a. Metacognitive and social cognition approaches to understanding the impact of schizophrenia on social quality of life. Psychiatry Research 2012;200(2-3):802-806.

39. Hasson-Ohayon I, Ehrlich-Ben Or S, Vahab K, Amiaz R, Weiser M, Roe D. Insight into mental illness and self-stigma: the mediating role of shame proneness. Psychiatry Res. 2012;200:802-6.

40. Hasson-Ohayon I, Kravetz S, Roe D, David AS, Weiser M. Insight into psychosis and quality of life. Compr Psychiatry. 2006;47:265-9. 